\title{
Conventional physical therapy and physical therapy based on reflex stimulation showed similar results in children with myelomeningocele
}

\author{
Fisioterapia convencional e fisioterapia com estimulação reflexa apresentaram \\ resultados semelhantes em crianças com mielomeningocele
}

Carolina Y. P. Aizawa', Mariana P. Morales' , Carolina Lundberg'1, Maria Clara D. Soares de Moura', Fernando C. G. Pinto', Mariana C. Voos' ${ }^{1}$ Renata H. Hasue'

\begin{abstract}
We aimed to investigate whether infants with myelomeningocele would improve their motor ability and functional independence after ten sessions of physical therapy and compare the outcomes of conventional physical therapy (CPT) to a physical therapy program based on reflex stimulation (RPT). Twelve children were allocated to CPT ( $n=6$, age 18.3 months) or RPT ( $n=6$, age 18.2 months). The RPT involved proprioceptive neuromuscular facilitation. Children were assessed with the Gross Motor Function Measure and the Pediatric Evaluation of Disability Inventory before and after treatment. Mann-Whitney tests compared the improvement on the two scales of CPT versus RPT and the Wilcoxon test compared CPT to RPT (before vs. after treatment). Possible correlations between the two scales were tested with Spearman correlation coefficients. Both groups showed improvement on self-care and mobility domains of both scales. There were no differences between the groups, before, or after intervention. The CPT and RPT showed similar results after ten weeks of treatment.
\end{abstract}

Keywords: meningomyelocele; physical and rehabilitation medicine; rehabilitation.

\section{RESUMO}

O estudo investigou se crianças com mielomeningocele melhorariam sua habilidade motora/funcional após dez sessões de fisioterapia e comparou o quadro motor de um grupo submetido à fisioterapia convencional (FC) com outro tratado com fisioterapia com estimulação reflexa (RF). Doze crianças foram alocadas em FC ( $n=6,18,3$ meses de idade) ou FR ( $n=6,18,2$ meses de idade). FR envolveu facilitação neuromuscular proprioceptiva. As crianças foram avaliadas com a Medida de Função Motora Grossa (GMFM) e o Inventário de Avaliação Pediátrica de Incapacidade (PEDI) antes e depois do tratamento. Testes de Mann-Whitney compararam a melhora na GMFM e PEDI (FC versus FR) e testes de Wilcoxon compararam FC e FR (antes versus depois). Possíveis correlações entre GMFM e PEDI foram testadas por coeficientes de Spearman. Ambos os grupos melhoraram na GMFM e PEDI (domínios autocuidado e mobilidade). Não houve diferença entre os grupos antes e após a intervenção. FC e FR apresentaram efeitos semelhantes depois de dez semanas de tratamento.

Palavras-chave: meningomeloocele; medicina física e reabilitação; reabilitação.

Myelomeningocele (MMC) is a form of spinal disraphism resulting from a defective closure of the posterior portion of the neural tube, during the fourth week of gestation $^{1}$. In Brazil, the incidence is 2.28:1,000 births ${ }^{1}$. An MMC frequently results in neuromusculoskeletal complications, including tetra- or paraparesis, neurogenic bowel and bladder, hydrocephalus and cognitive issues ${ }^{1,2,3}$. Many infants do not ambulate during infancy ${ }^{4,5,6}$ and have difficulty acquiring self-care skills ${ }^{2,5}$. Musculoskeletal immobility reflects on neuromuscular and cardiovascular systems and contributes to social and cognitive delays ${ }^{2,5}$.

Bartoneck and Saraste ${ }^{6}$ studied 53 children with MMC (3-11 years) to determine whether they achieved the expected level of ambulation. Thirty-one reached the expected ambulation based on their motor paresis. However, 22 performed worse than expected, due to balance disturbances and spasticity. Therefore, children with similar muscle paresis may exhibit different ambulatory function ${ }^{6}$. 
After this study, the same center followed 43 children prospectively, from six months to six years. Walking function was achieved at the one-year follow up in two children; at the two-year follow up in 14; at the four-year follow up in 28; and at the six-year follow up in 30 children. At the six-year follow-up, all children used orthoses, and nine had not achieved the level expected, based on muscle function ${ }^{7}$. Although nonambulating children had greater neurological and orthopedic symptoms, parents (including those of ambulating and non-ambulating children) reported a similar quality of life . $^{8}$

Trunk control is essential for the acquisition of a sitting position, which allows bimanual skills development ${ }^{9}$. The standing position is adopted passively, with orthotic devices, when the child does not have the strength to stand on his/her own $^{5,9}$. Conventional physical therapy (CPT) is usually performed with the aim of keeping the lower extremities aligned and to compensate for motor deficits in children with $\mathrm{MMC}^{9}$.

Prior studies have shown that lower limb weakness is related to worse ambulatory function ${ }^{10}$; however, strength training usually emphasizes the upper limbs and trunk. Although advances in multidisciplinary treatment of children with MMC have led to improvement in functional outcome, early clinical therapeutic interventions that might help infants acquire stronger or better functional control of their lower limbs have been neglected.

Ultrasound examinations of fetuses with MMC indicated the presence of hip and knee flexion and extension movements ${ }^{11}$. The presence of motor evoked potentials on the lower limbs suggests that there is excitable neural tissue below the level of malformation, including in the case of complete lower limb plegia ${ }^{12}$. The preservation of motor neurons can be explained by the fact that MMC results from the exposure of the dorsal part of the neural tube. Thus, the cells from the ventral horn of the spinal cord would be less affected, allowing lower limb motor activity.

Upper and lower limb synchrony patterns have also been described as lasting longer than isolated movements. Although this leg activity would be expected to increase in the weeks after birth, it usually decreases ${ }^{12}$. Taken together, these data indicate the presence of some neural activity in plegic muscles and the possibility of the preservation of sensory-motor spinal cord structures.

Considering that sensory and motor structures may be partially intact in babies with MMC, even when there is no function, it is possible that physical therapy could help not only in maintaining, but also in optimizing the sensory-motor function. Infants with MMC responded to the treadmill practice by stepping (but less so than infants with typical development) and showed increased motor activity, although they demonstrated a different developmental trajectory ${ }^{13}$. In this case, reflex stimulation, based on the Proprioceptive Neuromuscular Facilitation technique (PNF), could also be useful to improve motor function.

The PNF principles postulated that movement patterns, performed with facilitatory procedures (such as sensory information), would result in enhanced voluntary responses. The PNF technique was developed to strengthen muscles in the mass movement patterns in which they function. Patterns are spiral and diagonal and closely resemble functional movements in daily life. Manual contact facilitates underlying muscles and applies resistance to activate muscle spindles. When it is not possible to apply resistance, passive or assisted movements are recommended ${ }^{14}$. According to Voss, Ionta and Myers ${ }^{14}$, sensory stimulation provides the best possible motor response. Labyrinthine reflexes can be used to increase muscle tone and recruitment.

Sá, Santos and Xavier ${ }^{15}$ described a three-month program with 30-minute sessions, twice a week for children with cerebral palsy. One group was treated with PNF and another group with the Bobath technique. Children treated with PNF had greater sensory-motor improvement.

Britto, Correa and Vincent ${ }^{16}$ investigated the effect of PNF on muscle tone, low back pain, functional performance and gait speed in adults with myelopathy. They included two PNF techniques (rhythmic initiation and a combination of isotonics $)^{14}$ to facilitate rolling, sit-to-stand, walking and stair climbing. Patterns were rolling supine to lateral, transition from sitting to standing, standing on one leg, weight shifting forward and backward, walking forwards, backwards and sideways, going up and down stairs ${ }^{14}$. The PNF improved muscle tone, low back pain, functional performance and gait speed ${ }^{16}$.

No studies have tested the effects of physical therapy with reflex stimulation (RPT), based on PNF, in children with MMC and only two mention this approach in children with cerebral palsy ${ }^{15,17}$. Children with MMC might benefit from RPT, considering the evidence presented above $\mathrm{e}^{11,12,13}$. We hypothesized that RPT might provide experience and input relevant to, and usable by, the neuromotor systems of infants with incomplete spinal lesions.

Our goals were to 1 ) investigate whether infants with MMC would improve their functional activity (measured by the Gross Motor Function Measure) and/or functional independence (measured by the Pediatric Evaluation of Disability Inventory) after ten sessions of physical therapy; 2) compare the outcomes of CPT and RPT and 3) examine the relationship between functional independence and motor function.

\section{METHODS}

Twenty children diagnosed with MMC participated in this experimental study. They were separated into categories according to their MMC level. The Hoffer classification system ${ }^{18}$ was used to define lesion levels as thoracic (no sensation below the hips and no strength in hip muscles); upper lumbar (some sensation below the hips, some strength in hip adductors/flexors, or in knee extensors); lower lumbar (strength in knee flexors, ankle dorsiflexors, or hip abductors); and sacral (strength in ankle plantiflexors, or in hip extensors). 
The children were randomly enrolled into an experimental (RPT) or control group (CPT). This study was approved by the Ethics Committee of Faculdade de Medicina da Universidade de São Paulo (number 285/10, 09/29/2010). Parents gave written consent to the children to participate. Eight children did not complete the study, due to social difficulties (difficulties in taking public transportation) or clinical complications (infection).

All infants had hydrocephalus, with a ventriculoperitoneal shunt and urinary and fecal incontinence. The mean age was $18.2 \pm 15.6$ months in the RPT group and $18.3 \pm 12.4$ months in the CPT group.

Motor function was assessed by the Gross Motor Function Measure $^{19}$. Although the use of the Gross Motor Function Measure in children with spinal dysraphism has not been validated, it is the most-used scale in the literature. It has been used to measure the motor function of children with cerebral palsy, Down syndrome and spinal cord diseases ${ }^{20,21}$. The Gross Motor Function Measure consists of 88 items grouped in five dimensions: lying and rolling; sitting; crawling and kneeling; standing; walking, running and jumping. Items are scored on a four-point ordinal scale. Scores for each dimension are expressed as a percentage of the maximum score. A total score is obtained by adding the percentage of each dimension and dividing by five. Partial scores can also be calculated by adding the percent scores of two or more dimensions and dividing by the number of dimensions used ${ }^{19}$. In children who are unable to cooperate, scoring is based on the examiner's observation. The examiner can assist the child in assuming the initial testing position and observe movement patterns and the ability to overcome gravity.

The Pediatric Evaluation of Disability Inventory measures functional status in children between six months and seven-and-a-half years in three domains: self-care, mobility, and social function. It consists of an interview with the parents about the functional independence of the child. Numerous studies have examined the sensitivity of tests to identify changes during the recovery from brain or spinal injury ${ }^{5,22,23}$. In this study, we used the scaled scores of the Pediatric Evaluation of Disability Inventory.

\section{Intervention}

Children were randomly selected for the two treatment alternatives. We treated six children with RPT and six with CPT. Interventions consisted of ten 45-minute weekly sessions, performed by the same physical therapists. A blind examiner assessed all children before and after treatment.

The CPT interventions centered on optimizing mobility and maximizing independence. Sessions consisted of muscle strengthening, enhancing postural control and correcting positioning with orthotic devices. Muscle strengthening consisted of 10 to 30 repetitions of isotonic contractions of shoulder flexors, extensors, abductors, internal and external rotators, elbow flexors and extensors, with the child in a sitting position on the mat or chair. Trunk flexors and extensors were solicited with the child in a supine or prone position $^{23,24}$. Children who could not cooperate due to their age or cognitive impairment were assisted by the therapist (active-assisted mobility).

Postural control was trained by postural maintenance for as long as possible, (e.g. sitting, crawling, kneeling, standing), and postural changes (e.g. rolling, transition from supine to sitting, from prone to crawling, from sitting to crawling, from crawling to kneeling). Three to ten repetitions of each transition were performed in each session. Therapists helped children perform the transitions when necessary. Children unable to sit without support were not positioned kneeling. The correct positioning aimed to optimize motor function, and deformities were prevented with orthotic devices.

The RPT aimed to help the initiation of postural changes as well as the maintenance of different postures. Myotatic reflexes were obtained by muscle stretching before and during the contraction of the muscle belly or by tendon percussion. Skin receptors were stimulated by manual contact on the cutaneous region related to the specific muscle or motion desired, joint approximation or traction, and diagonal and rotational movement patterns ${ }^{14}$. Passive and assisted phases of rhythmic initiation stimulated rolling, sitting and crawling $^{14,16}$. Manual assistance was performed on a minimum of two muscles or muscle regions, with a minimum of five repetitions, in every session.

Righting reactions were used in the $\mathrm{RPT}^{14}$. Vestibular stimulation facilitated extensor muscles, and was performed by inclining the child to the side, starting from the upright sitting position. Three to five lateral inclinations were performed on each side. Children were positioned in prone on a ball and stimulated to resist the gravity force and, if possible, manual resistance of the therapist on the upper or lower trunk, to radiate muscle recruitment to the lower limbs. Movements were passive and assisted whenever possible $\mathrm{e}^{14,1516,17}$. Five to ten trunk extensions were performed. Attention was paid to the appropriate body position to facilitate the child's movement ${ }^{14}$.

Parents of children in both groups were taught home exercises programs, based on the training during therapy sessions. They were encouraged to practice the exercises for 15 to 20 minutes daily.

\section{Statistical analysis}

Non-parametrical tests were used due to the small number of children in each group and to non-normally distributed data. Mann-Whitney tests investigated possible differences in the improvement in the Gross Motor Function Measure and the Pediatric Evaluation of Disability Inventory between the groups (CPT and RPT). Wilcoxon tests compared the performance of each group before after the treatment. Spearman's correlation tests investigated possible relationships between the Gross Motor Function Measure and Pediatric Evaluation of Disability Inventory scores. The significance level was 0.05 . 


\section{RESULTS}

As most patients in both groups scored zero on initial and final assessments for standing and walking, running, and jumping on the Gross Motor Function Measure, the score ABC [(sum of domains A, B and C)/3] was used. Table 1 shows the children's characteristics and scores.

In the CPT group, domain A of the Gross Motor Function Measure improved from 91.6 to 94.1 (2.5\%) and domain
B from 63.7 to 80.0 (16.3\%). In the RPT group, domain A improved from 71.6 to 89.6 (18.0\%) and domain B improved from 37.5 to 61.7 (24.2\%).

Individual findings of the scaled scores on the Pediatric Evaluation of Disability Inventory before and after treatment are shown in Table 2. Wilcoxon tests compared the performances before and after treatment for each group. Both groups showed significant improvement on all items, except on social function (Table 3).

Table 1. Gender, age, neurological level of myelomeningocele and respective scores (in percentage) on Gross Motor Function Measure of each child treated with RPT or CPT obtained on assessments 1 (before treatment) and 2 (after treatment).

\begin{tabular}{|c|c|c|c|c|c|c|c|c|c|c|c|c|c|c|c|c|c|}
\hline \multirow{2}{*}{$\begin{array}{l}\text { Group/ } \\
\text { subject }\end{array}$} & \multirow{2}{*}{ Gender } & \multirow{2}{*}{$\begin{array}{c}\text { Age } \\
\text { (months) }\end{array}$} & \multirow{2}{*}{$\begin{array}{c}\text { Neurologial } \\
\text { level }\end{array}$} & \multicolumn{7}{|c|}{ Assessment 1} & \multicolumn{7}{|c|}{ Assessment 2} \\
\hline & & & & A & B & C & $\mathrm{D}$ & $E$ & $A B C$ & Total & A & B & $C$ & D & $E$ & $A B C$ & Total \\
\hline \multicolumn{18}{|c|}{ RPT } \\
\hline C. & $\mathrm{m}$ & 22 & High lumbar & 73.0 & 42.0 & 12.0 & 0.0 & 0.0 & 42.0 & 25.0 & 89.0 & 70.0 & 20.0 & 5.0 & 0.0 & 59.0 & 27.0 \\
\hline J. & $\mathrm{m}$ & 48 & High lumbar & 70.6 & 35.0 & 7.1 & 0.0 & 0.0 & 37.0 & 22.0 & 88.2 & 53.3 & 7.1 & 0.0 & 0.0 & 49.0 & 29.0 \\
\hline N. & $f$ & 7 & Low lumbar & 51.0 & 28.3 & 0.0 & 0.0 & 0.0 & 26.0 & 15.0 & 72.5 & 38.3 & 0.0 & 0.0 & 0.0 & 36.0 & 22.0 \\
\hline K. & $\mathrm{m}$ & 7 & Low lumbar & 64.7 & 16.7 & 0.0 & 0.0 & 0.0 & 27.0 & 16.0 & 96.1 & 71.7 & 0.0 & 0.0 & 0.0 & 55.0 & 33.0 \\
\hline L. & $f$ & 10 & Sacral & 72.5 & 40.0 & 0.0 & 0.0 & 0.0 & 37.0 & 22.0 & 90.2 & 53.3 & 2.4 & 2.6 & 0.0 & 48.0 & 29.0 \\
\hline J. & $f$ & 20 & Sacral & 100.0 & 88.5 & 57.1 & 5.1 & 0.0 & 82.0 & 50.0 & 100.0 & 91.7 & 62.0 & 10.3 & 5.6 & 84.0 & 54.0 \\
\hline Median & & & & 71.6 & 37.5 & 3.6 & 0.0 & 0.0 & 37.0 & 22.0 & 89.6 & 61.7 & 4.8 & 1.3 & 0.0 & 52.0 & 29.0 \\
\hline \multicolumn{18}{|c|}{ CPT } \\
\hline A. & $\mathrm{m}$ & 3 & High lumbar & 19.6 & 15.0 & 0.0 & 0.0 & 0.0 & 11.0 & 7.0 & 49.0 & 23.3 & 0.0 & 0.0 & 0.0 & 24.0 & 14.0 \\
\hline G. & $\mathrm{m}$ & 37 & High lumbar & 88.2 & 75.0 & 21.4 & 0.0 & 0.0 & 61.0 & 36.0 & 88.2 & 85.0 & 21.4 & 0.0 & 0.0 & 65.0 & 39.0 \\
\hline I. & f & 6 & Low lumbar & 29.4 & 18.3 & 0.0 & 0.0 & 0.0 & 16.0 & 9.0 & 70.6 & 31.7 & 0.0 & 0.0 & 0.0 & 34.0 & 20.0 \\
\hline $\mathrm{L} ;$ & $\mathrm{m}$ & 20 & Low lumbar & 100.0 & 81.7 & 0.0 & 0.0 & 0.0 & 60.0 & 36.0 & 100.0 & 86.7 & 62.0 & 5.1 & 1.3 & 82.0 & 51.0 \\
\hline N. & $f$ & 21 & Sacral & 95.0 & 52.3 & 10.0 & 0.0 & 0.0 & 52.0 & 31.0 & 100.0 & 75.0 & 10.0 & 10.0 & 0.0 & 61.0 & 39.0 \\
\hline M. & $f$ & 24 & Sacral & 98.0 & 77.9 & 38.1 & 0.0 & 0.0 & 71.0 & 42.0 & 100.0 & 86.7 & 54.8 & 10.3 & 1.4 & 80.0 & 50.0 \\
\hline Median & & & & 91.6 & 63.7 & 5.0 & 0.0 & 0.0 & 56.0 & 33.5 & 94.1 & 80.0 & 15.7 & 2.6 & 0.0 & 63.0 & 39.0 \\
\hline
\end{tabular}

RPT: reflex physical therapy group;CPT: conventional physical therapy group;f: female; $m$ : male; ABC Score: mean score (in percentage) of domains A, B and C of the gross motor function measure; total score: mean score (in percentage) of all domains of the gross motor function measure.

Table 2. Gender, age, neurological level of myelomeningocele and respective scaled scores on functional skills of the Pediatric Evaluation of Disability Inventory (PEDI) of each child treated with RPT or CPT obtained on assessments 1 (before treatment) and 2 (after treatment).

\begin{tabular}{|c|c|c|c|c|c|c|c|c|c|}
\hline \multirow{2}{*}{$\begin{array}{l}\text { Group/ } \\
\text { subject }\end{array}$} & \multirow{2}{*}{ Gender } & \multirow{2}{*}{$\begin{array}{c}\text { Age } \\
\text { (months) }\end{array}$} & \multirow{2}{*}{$\begin{array}{c}\text { Neurologial } \\
\text { level }\end{array}$} & \multicolumn{3}{|c|}{ Assessment 1} & \multicolumn{3}{|c|}{ Assessment 2} \\
\hline & & & & SC & MO & SF & SC & MO & SF \\
\hline \multicolumn{10}{|c|}{ RPT } \\
\hline C. & $\mathrm{m}$ & 22 & High lumbar & 34.1 & 11.4 & 31.6 & 44.4 & 15.2 & 42.5 \\
\hline J. & $\mathrm{m}$ & 48 & High lumbar & 37.8 & 30.6 & 14.7 & 39.6 & 32.0 & 34.0 \\
\hline N. & $f$ & 7 & Low lumbar & 29.4 & 6.1 & 35.1 & 34.1 & 11.4 & 35.1 \\
\hline K. & $\mathrm{m}$ & 7 & Low lumbar & 28.0 & 11.4 & 31.6 & 42.0 & 18.2 & 42.5 \\
\hline L. & $f$ & 10 & Sacral & 34.1 & 11.4 & 21.6 & 44.4 & 15.2 & 45.0 \\
\hline J. & $f$ & 20 & Sacral & 39.6 & 32.0 & 39.6 & 45.2 & 35.9 & 46.2 \\
\hline Median & & & & 34.1 & 11.4 & 31.6 & 43.2 & 16.7 & 42.5 \\
\hline \multicolumn{10}{|c|}{ СРT } \\
\hline A. & $\mathrm{m}$ & 3 & High lumbar & 11.8 & 0.0 & 14.7 & 26.2 & 6.1 & 14.7 \\
\hline G. & $\mathrm{m}$ & 37 & High lumbar & 41.2 & 32.0 & 47.9 & 48.9 & 33.4 & 56.0 \\
\hline ।. & f & 6 & Low lumbar & 21.4 & 6.1 & 27.2 & 26.2 & 11.4 & 30.0 \\
\hline L; & $\mathrm{m}$ & 20 & Low lumbar & 39.6 & 34.7 & 41.8 & 45.2 & 40.3 & 41.8 \\
\hline N. & $f$ & 21 & Sacral & 39.6 & 32.0 & 41.8 & 45.2 & 33.4 & 41.8 \\
\hline M. & $f$ & 24 & Sacral & 50.3 & 33.4 & 47.9 & 56.2 & 47.0 & 53.7 \\
\hline Median & & & & 39.6 & 32.0 & 41.8 & 45.2 & 33.4 & 41.8 \\
\hline
\end{tabular}

RPT: reflex physical therapy group; CPT: conventional physical therapy group; f: female; m: male; SC: self-care; MO: mobility; SF: social function. 
The Mann-Whitney U test showed no significant difference between the groups on the Gross Motor Function Measure (total score: $U=9.0, p=0.54$; score ABC: $U=11.0$, $\mathrm{p}=0.84$ ). The Mann-Whitney $U$ test also showed no significant difference on the Pediatric Evaluation of Disability Inventory (self-care: $\mathrm{U}=9.5, \mathrm{p}=0.55$; mobility: $\mathrm{U}=8.0, \mathrm{p}=0.42$; social function: $U=5.0, p=0.15$ ).

A possible relation between the two scales (Gross Motor Function Measure and Pediatric Evaluation of Disability Inventory) was investigated with Spearman's non-parametric tests. Since there were no differences between the groups, all twelve of the patients were analyzed together. There were strong correlations between the Gross Motor Function Measure and Pediatric Evaluation of Disability Inventory scores (Table 4).

\section{DISCUSSION}

Two treatment protocols for children with MMC were compared: CPT and RPT. In both groups, postural control improved, mainly in prone, supine and sitting. The CPT group probably improved this control with adaptations (joint stabilization with orthoses ${ }^{23,24}$, range of motion maintenance ${ }^{23}$ ) and compensations (stimulation of movements and weight bearing with lower $\operatorname{limbs}^{23}$ ). Training was primarily directed to strengthen and optimize the preserved

Table 3. Results of the Wilcoxon tests. The score on assessment 2 (after treatment) was compared to assessment 1 (before treatment).

\begin{tabular}{lccccc}
\hline Variable & GMFM_T & GMFM_ABC & PEDI_SC & PEDI_MO & PEDI_SF \\
\hline RPT & & & & & \\
p-value & 0.04 & 0.04 & 0.04 & 0.04 & 0.06 \\
CPT & & & & & \\
p-value & 0.04 & 0.04 & 0.04 & 0.04 & 0.10 \\
\hline
\end{tabular}

RPT: reflex physical therapy; CPT: conventional physical therapy; GMFM_T: total score on GMFM; GMFM_ ABC: score on domains A, B and C of GMFM [(domain A+ domain B+ domain C) /3]; PEDI: pediatric evaluation of disability inventory; PEDI_SC: self-care section of PEDI; PEDI_MO: mobility section of PEDI; PEDI_SF: social function section of PEDI. sensorimotor functions ${ }^{24}$ and took advantage of cognitive abilities, stimulating the child to cooperate during the exercises whenever possible.

The RPT aimed to facilitate the conduction of stimuli from the extremities to the cortex by the remaining afferent pathways. In the RPT group, there was possibly an increase of peripheral sensory information. This increase in sensory information arriving at the cortex is important to improve sensory-motor integration and the quality of motor responses. ${ }^{25}$ Conversely, improvement in muscular recruitment could explain gains in postural changes after CPT. Reflex physical therapy can be performed without the cooperation of the patient and may be a good alternative in patients 0-2 years, or for patients with cognitive impairment.

No significant differences were found between CPT and RPT. It is possible that CPT optimized motor strategies of the trunk and upper limbs to compensate for the loss of lower limb control. Conversely, RPT could favor better lower limb control. Both protocols may have resulted in higher activation of the remaining spinal interneurons, or of cortical neuronal networks. It is interesting to note that, although we analyzed a relatively short period of treatment (10 weeks), we detected significant improvement in both groups.

According to Muir and Steeves ${ }^{26}$ and Raineteau and $\mathrm{Schwab}^{27}$, the improvement of functional recovery after spinal injuries depends on reorganization of undamaged neural pathways. This could enhance the limited ability of neurons to restore damaged connections between the spinal cord and brain $^{26,27}$. Spinal cord circuits are capable of significant reorganization induced by both activity-dependent and injuryinduced plasticity. Regenerating spinal tract fibers needs functional training to make the appropriate connections, and training effects are enhanced by regenerating fibers ${ }^{25}$. This would result, for instance, in a better impulsion for rolling, or in a better fixation of the lower limbs during the transition from lying to sitting. In fact, as shown in Table 1, these skills of the Gross Motor Function Measure improved $2.5 \%$ in the CPT on domain A, which assesses lying and rolling, and $16.3 \%$ on domain B, which assesses sitting. In the RPT, these skills improved $18.0 \%$ on domain A and $24.2 \%$ on domain B.

Table 4. Results of the non-parametrical Spearman's correlations tests. There were strong correlations between parts A and B and average ABC of Gross Motor Function Measure (GMFM) and the sections of total scores of Pediatric Evaluation of Disability Inventory (PEDI).

\begin{tabular}{|c|c|c|c|c|c|c|}
\hline \multirow{2}{*}{ Variable } & \multicolumn{3}{|c|}{ Assessment 1 (before treatment) } & \multicolumn{3}{|c|}{ Assessment 2 (after treatment) } \\
\hline & GMFM_A & GMFM_B & GMFM_ABC & GMFM_A & GMFM_B & GMFM_ABC \\
\hline PEDI_SC & 0.87 & 0.87 & 0.86 & 0.86 & 0.90 & 0.92 \\
\hline PEDI_MO & 0.96 & 0.92 & 0.94 & 0.91 & 0.94 & 0.94 \\
\hline PEDI_SF & 0.84 & 0.84 & 0.82 & 0.77 & 0.84 & 0.87 \\
\hline PEDI_TOTAL & 0.88 & 0.88 & 0.86 & 0.86 & 0.90 & 0.93 \\
\hline
\end{tabular}

GMFM_A: score on domain A of GMFM; GMFM_B: score on domain B of GMFM; GMFM_ABC: score on domains A, B and C of GMFM [(domain A + domain B + domain C)/3]; PEDI_SC: self-care section of PEDI; PEDI_MO: mobility section of PEDI; PEDI_SF: social function section of PEDI; PEDI_TOTAL: total score of functional skills parts of PEDI. 
Plasticity after spinal cord injury can be initiated by specific patterns of sensory feedback, leading to a reorganization of spinal and cortical networks ${ }^{26}$. Many rehabilitation approaches focus on the exploitation of spinal cord plasticity below the level of lesion, for example, by locomotor training. Protocols with spinal injury patients showed that training can improve the functional locomotor abilities. This plasticity becomes even more evident in the ability of spinalized animals to regain a certain degree of motor function ${ }^{25}$. Additionally, Pantall et al. ${ }^{28}$ verified that increased sensory feedback improved the step pattern of children with MMC who walked on a motorized treadmill.

Recently, Heathcock et al. ${ }^{29}$ proposed treadmill training for toddlers with spinal cord injury before the onset of walking. They reported the intervention and stepping behaviors, on a treadmill and on the ground, of a toddler after the surgical removal of a rare spinal tumor resulting in spinal cord injury. The toddler presented with an inability to step on the left, rare stepping on the right, and an apparent lack of sensation in the lower extremities. Step training on a treadmill and on the ground occurred once a week from 15 to 35 months of age. Independent symmetrical stepping emerged both on and off the treadmill over 20 months. Walking speed increased, and milestones important to ground walking developed. Independent steps developed during the intervention with little sensory and motor development of the lower extremities during the first year of life $\mathrm{e}^{29}$.

Very few studies discuss the dosing of physical therapy for MMC treatment. Karmel-Ross et al. ${ }^{30}$ reported the positive effect of electrical stimulation applied to the quadriceps femoris in conjunction with walking and standing activities for 30 minutes sessions, six times per week for eight weeks ${ }^{30}$. The present study reached positive results in 45 minutes per week for 10 weeks. Also in our study, both groups' parents were taught an individualized program of home exercises and encouraged to practice them for 15 to 20 minutes daily.

The present study shows strong correlations between the Gross Motor Function Measure and the Pediatric Evaluation of Disability Inventory scores for both groups. We believe that the improvement in motor function resulted in a higher functional independence. Danielsson et al. ${ }^{5}$ found reduced muscle strength and occurrence of spasticity around the hip and knee affected ambulation, functional mobility and self-care (measured by the Pediatric Evaluation of Disability Inventory). They also concluded that patients with reduced functional mobility and self-care experienced a lower physical quality of living. The Gross Motor Function Measure scores may indirectly reflect muscle strength and spasticity. As a limitation of the present study, we must mention that we did not measure the amount of tactile or proprioceptive stimulation of the lower limbs reaching the cortex. However, previous studies have reported that part of these pathways is intact in many patients ${ }^{11,12}$. Besides, both protocols offered vestibular stimuli and all kinds of sensory stimuli in the upper limbs, which could have helped sensory-motor integration in the spine and cortex.

Another limitation is that the scales used in the present study do not allow the differentiation of automatic and voluntary control, nor the differentiation of upper limb, lower limb, or trunk movements. We believe that the global automatic and voluntary controls have improved, because the performance on tasks that involve weight bearing, such as rolling and crawling showed improvement.

We must also mention that the groups had a small number of patients with heterogeneous clinical features due to the low prevalence of MMC. Furthermore, a control group (with no intervention) was not compared, because we believe it would be unethical to follow a group with no therapy. We must also consider age perspective in a child's development over ten weeks, which may have influenced our results.

In conclusion, two different physical therapy protocols resulted in motor and functional improvement in children with MMC. The gains in motor ability were associated with a better functional status. Further studies are necessary to improve physical therapy techniques for children with MMC as well as to verify the benefits of physical therapy based on the PNF concept in the treatment of children with neurological dysfunctions.

\section{References}

1. Zambelli H, Carelli E, Honorato D, Marba S, Coelho G, Carnevalle A et al. Assessment of neurosurgical outcome in children prenatally diagnosed with myelomeningocele and development of a protocol for fetal surgery to prevent hydrocephalus. Childs Nerv Syst. 2007;23(4):421-5. https://doi.org/10.1007/s00381-006-0261-x

2. Schoenmakers MA, Uiterwaal CS, Gulmans VA, Gooskens RH, Helders PJ. Determinants of functional independence and quality of life in children with spina bifida. Clin Rehabil. 2005;19(6):677-85. https://doi.org/10.1191/0269215505cr865oa

3. Warf BC. Hydrocephalus associated with neural tube defects: characteristics, management, and outcome in subSaharan Africa. Childs Nerv Syst. 2011;27(10):1589-94. https://doi.org/10.1007/s00381-011-1484-z

4. Seitzberg A, Lind M, Biering-Sørensen F. Ambulation in adults with myelomeningocele. Is it possible to predict the level of ambulation in early life? Childs Nerv Syst. 2008;24(2):231-7. https://doi.org/10.1007/s00381-007-0450-2

5. Danielsson AJ, Bartonek A, Levey E, McHale K, Sponseller $P$, Saraste H. Associations between orthopaedic findings, ambulation and health-related quality of life in children with myelomeningocele. J Child Orthop. 2008;2(1):45-54. https://doi.org/10.1007/s11832-007-0069-6

6. Bartonek A, Saraste H. Factors influencing ambulation in myelomeningocele: a cross-sectional study. Dev Med Child Neurol. 2001;43(4):253-60. https://doi.org/10.1017/S0012162201000482

7. Bartonek A. Motor development toward ambulation in preschool children with myelomeningocele: a prospective study. Pediatr Phys Ther. 2010;22(1):52-60. https://doi.org/10.1097/PEP.0b013e3181cc132b 
8. Bartonek A, Saraste H, Danielsson A. Health-related quality of life and ambulation in children with myelomeningocele in a Swedish population. Acta Paediatr. 2012;101(9):953-6. https://doi.org/10.1111/j.1651-2227.2012.02742.x

9. McDonald CM, Jaffe KM, Mosca VS, Shurtleff DB. Ambulatory outcome of children with myelomeningocele: effect of lower-extremity muscle strength. Dev Med Child Neurol. 1991;33(6):482-90. https://doi.org/10.1111/j.1469-8749.1991.tb14913.x

10. Agre JC, Findley TW, McNally MC, Habeck R, Leon AS, Stradel L et al. Physical activity capacity in children with myelomeningocele. Arch Phys Med Rehabil. 1987;68(6):372-7.

11. Warsof SL, Abramowicz JS, Sayegh SK, Levy DL. Lower limb movements and urologic function in fetuses with neural tube and other central nervous system defects. Fetal Ther. 1988;3(3):129-34. https://doi.org/10.1159/000263344

12. Sival DA, van Weerden TW, Vles JS, Timmer A, Dunnen WF, Staal-Schreinemachers AL et al. Neonatal loss of motor function in human spina bifida aperta. Pediatrics. 2004;114(2):427-34. https://doi.org/10.1542/peds.114.2.427

13. Teulier C, Smith BA, Kubo M, Chang CL, Moerchen V, Murazko K et al. Stepping responses of infants with myelomeningocele when supported on a motorized treadmill. Phys Ther. 2009;89(1):60-72. https://doi.org/10.2522/ptj.20080120

14. Voss DE, Ionta MK, Myers BJ. Facilitação neuromuscular proprioceptiva. São Paulo: Médica Panamericana;1987.

15. Sá CSC, Santos FH, Xavier GF. Motor, sensorial and cognitive changes in children with spastic diparetic cerebral palsy submitted to Kabat and Bobath physiotherapy approaches. Rev Fisioter Univ São Paulo. 2004;11(1):56-65

16. Britto VLS, Correa R, Vincent MB. Proprioceptive neuromuscular facilitation in HTLV-I-associated myelopathy/tropical spastic paraparesis. Rev Soc Bras Med Trop. 2014;47(1):24-9. https://doi.org/10.1590/0037-8682-0245-2013

17. Levitt S. Proprioceptive neuromuscular facilitation techniques in cerebral palsy. Physiotherapy. 1966;52(2):46-51.

18. Hoffer MM, Feiwell E, Perry R, Perry J, Bonnett C. Functional ambulation in patients with myelomeningocele. J Bone Joint Surg Am. 1973;55(1):137-48. https://doi.org/10.2106/00004623-197355010-00014

19. Russell DJ, Rosenbaum PL, Lane M, Gowland C, Goldsmith CH, Boyce WF et al. Training users in the gross motor function measure: methodological and practical issues. Phys Ther. 1994;74(7):630-6.
20. Adair B, Said CM, Rodda J, Morris ME. Psychometric properties of functional mobility tools in hereditary spastic paraplegia and other childhood neurological conditions. Dev Med Child Neurol. 2012;54(7):596-605. https://doi.org/10.1111/j.1469-8749.2012.04284.x

21. Wang HY, Yang YH, Jong YJ. Correlations between change scores of measures for muscle strength and motor function in individuals with spinal muscular atrophy types 2 and 3. Am J Phys Med Rehabil. 2013;92(4):335-42. https://doi.org/10.1097/PHM.0b013e318269d66b

22. Tsai PY, Yang TF, Chan RC, Huang PH, Wong TT. Functional investigation in children with spina bifida: measured by the Pediatric Evaluation of Disability Inventory (PEDI). Childs Nerv Syst. 2002;18(1-2):48-53. https://doi.org/10.1007/s00381-001-0531-6

23. Choksi A, Townsend EL, Dumas HM, Haley SM.

Functional recovery in children and adolescents with spinal cord injury. Pediatr Phys Ther. 2010;22(2):214-21. https://doi.org/10.1097/PEP.0b013e3181dc011a

24. McDonald CM. Rehabilitation of children with spinal dysraphism. Neurosurg Clin N Am. 1995;6(2):393-412.

25. Dietz V. G. Heiner Sell memorial lecture: neuronal plasticity after spinal cord injury: significance for present and future treatments. J Spinal Cord Med. 2006;29(5):481-8. https://doi.org/10.1080/10790268.2006.11753897

26. Muir GD, Steeves JD. Sensorimotor stimulation to improve locomotor recovery after spinal cord injury. Trends Neurosci. 1997;20(2):72-7. https://doi.org/10.1016/S0166-2236(96)10068-0

27. Raineteau O, Schwab ME. Plasticity of motor systems after incomplete spinal cord injury. Nat Rev Neurosci. 2001;2(4):263-73. https://doi.org/10.1038/35067570

28. Pantall A, Teulier C, Smith BA, Moerchen V, Ulrich BD. Impact of enhanced sensory input on treadmill step frequency: infants born with myelomeningocele. Pediatr Phys Ther. 2011;23(1):42-52. https://doi.org/10.1097/PEP.0b013e318206eefa

29. Heathcock JC, Christensen C, Bush K, Butler M, Buehner $\mathrm{JJ}$, Basso DM. Treadmill training after surgical removal of a spinal tumor in infancy. Phys Ther. 2014;94(8):1176-85. https://doi.org/10.2522/ptj.20130508

30. Karmel-Ross, K.,Cooperman,D.R.,Van Doren, C. L. The effect of electrical stimulation on quadriceps femoris muscle torque in children with spina bifida. Phys Ther. 1992;72(10):723-30. 